MATEC Web of Conferences 22,01014 (2015)

DOI: $10.1051 /$ matec conf/ 20152201014

(C) Owned by the authors, published by EDP Sciences, 2015

\title{
Performance Assessment of Flight Simulator Servo System Based on LQG Performance Benchmark
}

\author{
Huibo Liu, Hao Wu \& Mu Liu \\ School of Information Engineering, Inner Mongolia University of Science and Technology, Baotou, Inner Mon- \\ golia, China
}

\begin{abstract}
Flight simulator is an important application in the field of aerospace as semi-physical simulation equipment. As it requires supreme control precision and stability, it is especially important to search the performance assessment of flight simulator servo system. The traditional researches on flight simulator control performance index is more about dynamic output tracking features but few on input characteristics and effects. Based on Linear Quadratic Gaussian (LQG) performance benchmark, this paper makes analyses on high precision flight simulator in three kinds of controller while considering the influences of input and output signals' effect on controllers. After processing the input and output data, combined with the linear fitting method, we can obtain LQG performance tradeoff curve. Through comparing the controller's actual performance with the optimal performance, we'll gain the controller's control performance index and its potential.
\end{abstract}

Keywords: Performance Assessment; LQG; flight simulator servo system; Potential performance index

\section{INTRODUCTION}

The flight simulator servo system is a semi-physical simulation equipment in aerospace field where the design and development of aerospace aircraft, missiles play key roles. The simulator's research is of great significance to the national defense science and technology. All along, the simulator's research focuses on the advanced control algorithm, and the assessment of its performance is obviously insufficient.

Actually, since Harris (1989) [1] published minimum variance performance benchmark, performance assessment theory had been enriched. Control Performance Assessment (CPA) technology already ensures those in the whole process of industrial production of various controllers can run at good states. Control Performance Assessment's goal is to estimate a lower bound performance of the designed control system by monitoring the running system and processing a range of data getting from the closed-loop system. With certain indexes, operators can judge the running state of the system and figure out whether the current control performance accords with the anticipated design goal. If it cannot meet the design requirements, CPA will locate the fault point in time and give reasonable suggestions.

In the control system of the assessment theory, Minimum Variance Control (MVC) and Generalized Minimum Variance (GMV) are commonly used with performance indexes [2]. Among these methods, MVC benchmark has relatively wide applications because of its characteristics of non-invasive in single variable and closed-loop data. However, MVC benchmark in the control system only gives the minimum variance of the output, and it cannot reflect the impact of inputs on the whole control system. As for the performance assessment of fast response systems which are similar to supreme precision flight simulator servo system, minimum variance benchmark cannot fully meet the requirements. Based on LQG benchmark, this paper considers the effects of input and output variances on the whole control system.

\section{EVALUATION ON THE LQG BENCHMARK PERFORMANCE}

\subsection{The trade-off curve and control performance benchmark}

In the actual control, engineers cannot simply think that the control system meets the requirements as long as output variance reaches performance requirements. In many cases, the designer should not only ensure the output variance to be minimum but also hope that the input control in a certain range, so reflecting the input variance effects on the control system factually is particularly important. Based on the former MVC benchmark, a further promotion-Kadali \& Huang (2002) [3] put forward the LQG benchmark.

The LQG benchmark is to solve such a problem:

If $E\left[\mathrm{u}_{t}^{2}\right] \leq \alpha$, what is $\min E\left[\mathrm{y}_{t}^{2}\right]$ ?

By solving the LQG problem, we can obtain control performance tradeoff curve. Define LQG objective function as:

$$
J[\lambda]=E\left[y_{t}^{2}\right]+\lambda E\left[\mathbf{u}_{t}^{2}\right]
$$

Taking different values to $\lambda$, different sets of optimal solutions of $E\left[u_{t}^{2}\right]_{\text {and }} E\left[\mathrm{y}_{t}^{2}\right]_{\text {can be calcu- }}$ 


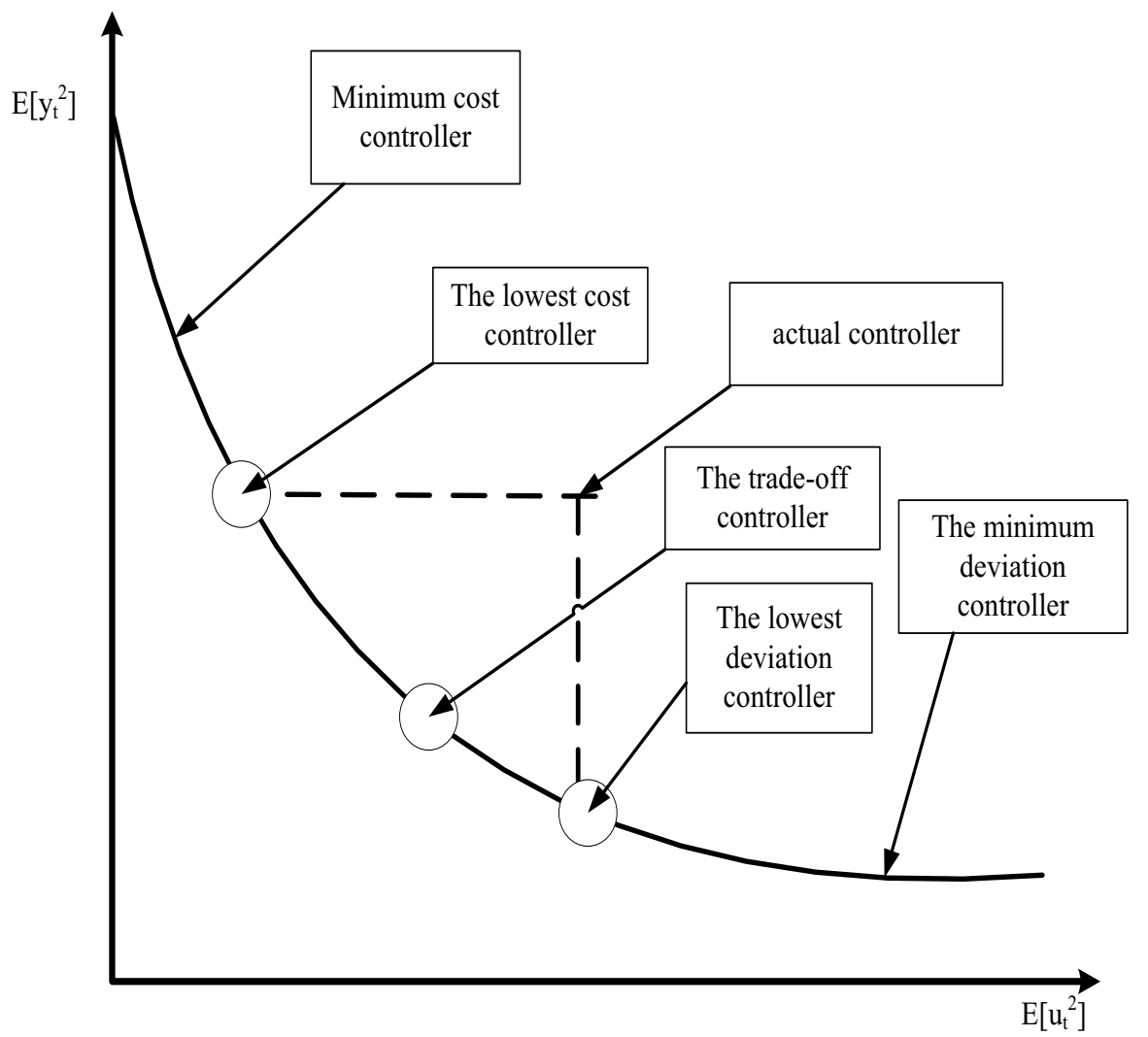

Figure 1. The LQG trade-off curve and five kinds of optimal controllers

lated, and then take $E\left[\boldsymbol{u}_{t}^{2}\right]$ as abscissa, $E\left[\mathrm{y}_{t}^{2}\right]_{\text {as }}$ ordinate, and translate the optimal solutions into coordinate graphs. The control performance tradeoff curve is as shown in Figure 1.

As shown in Figure 1, what the five optimal controllers on the LQG trade-off curve represent are five benchmarks upon different controlled objectives. These controllers usually show theoretical lower bounds, which are not able to be reached easily in practice. In practical applications, we generally only use one of them to evaluate one certain system's control performance [4]. The concrete implications of these controllers are as follows:

1) The minimum cost control: At the leftmost of the trade-off curve. Minimum cost control provides a non-compensating control performance in the condition of the minimum cost

2) The lowest cost control: This optimal controller can achieve the same output error with current controller using the theoretically lowest cost.

3) The lowest deviation control: With the same controlling cost and existing conditions, this method can reach the minimum output deviation.

4) The trade-off control: This is a compromise choice between the lowest cost and the minimum deviation; it can be obtained by drawing a vertical line in the trade-off curve of the present controller.

5) The minimum deviation control: At the rightmost of the trade-off curve. Minimum deviation controller provides the minimum possible deviation theoretically.

\subsection{Performance evaluation of $L Q G$ benchmark}

It can be seen from the analysis above, if we want to evaluate the performance based on LQG benchmark, getting the LQG curve should be the first priority [5]. According to the degree of difficulty of establishing a model of a control system, there are three situations: when the control system model can be established accurately, we can employ state space model algorithm or Generalized Predictive Control (GPC) algorithm; when the system model is ARMAX model, we can use polynomial algorithm; closed-loop subspace method can be used when it is difficult to determine accurate model. Because using the method of closed-loop subspace identification does not need to identify the complete object model, we do not have to conduct complex calculations, and the data of input and output can help us to establish subspace matrix, and it also reduces errors in the complex calculation. 
2.2.1 Design of LQG controller

Linear time-invariant system's state space model is described as follows:

$x_{k+1}=A x_{k}+B u_{k}+K e_{k}$

$y_{k}=C x_{k}+D u_{k}+e_{k}$

The state variables $x(k) \in \mathrm{R}^{\mathrm{n}}$, the output variables $y(k) \in \mathrm{R}^{l}$, control variables $u(k) \in \mathrm{R}^{\mathrm{m}}$ in the formulas, $e_{k}$ is Gauss noise, $K$ is the Calman steady state gain matrix. By collecting the input and output data as well as using subspace identification algorithm, the predicted system model can be established:

$y_{f}=L_{w} w_{p}+L_{u} u_{f}+L_{e} e_{f}$

$p$ presents the past time, and $f$ presents the future time. $L_{w}, L_{u}$, and $L_{e}$ are the subspace matrixes, respectively presenting corresponding state, deterministic input, random input. Obtained from the QR decomposition:

$$
\begin{aligned}
& y_{f}=\left[\begin{array}{ll}
\mathrm{y}(t+1), \mathrm{y}(t+2) \ldots, & \mathrm{y}(t+n)
\end{array}\right]^{T} \\
& u_{f}=\left[\begin{array}{ll}
\mathrm{u}(t+1), \mathrm{u}(t+2) \ldots, \mathrm{u}(t+n)
\end{array}\right]^{T} \\
& y_{p}=\left[\begin{array}{lll}
y(t-n+1), y(t-N+2) \ldots, & y(t)
\end{array}\right]^{T} \\
& u_{p}=\left[\begin{array}{ll}
u(t-n+1), u(t-N+2) \ldots, & u(t)
\end{array}\right]^{T} \\
& w_{p}=\left(\mathrm{y}_{p} ; \mathrm{u}_{p}\right)
\end{aligned}
$$

The objective function of LQG controller is designed as:

$$
\begin{aligned}
& J_{L Q G}=E\left\{\sum _ { k = 1 } ^ { N } \left[(\mathrm{y}(\mathrm{t}+\mathrm{k})-\mathrm{r}(\mathrm{t}+\mathrm{k}))^{T} \mathrm{~W}_{y}(\mathrm{y}(\mathrm{t}+\mathrm{k})\right.\right. \\
& \left.\left.-\mathrm{r}(\mathrm{t}+\mathrm{k}))+\lambda u(\mathrm{t}+\mathrm{k})^{T} \mathrm{~W}_{u} \mathrm{u}(\mathrm{t}+\mathrm{k})\right]\right\}
\end{aligned}
$$

$E$ represents the expectation operator, $W_{y}$ and $W_{u}$ respectively represent weights of control part and output part in variables, $\mathrm{y}(\mathrm{t}+\mathrm{k})$ represents the time output, $\mathrm{r}(\mathrm{t}+\mathrm{k})$ represents the same time setting value, and $\lambda$ is used to change the weights. Put the formula (2) into (3), we can get LQG objective function based on subspace model:

$J_{L Q G}=\left(L_{w} \mathrm{w}_{p}+\mathrm{L}_{u} \mathrm{u}_{f}-\mathrm{r}_{f}\right)^{T} \times \mathrm{W}_{y}\left(L_{w} \mathrm{w}_{p}+\mathrm{L}_{u} \mathrm{u}_{f}-\mathrm{r}_{f}\right)$

$+\lambda u_{f}^{T} \mathrm{~W}_{u} \mathrm{u}_{f}$

According to the objective function above, it meets the condition:

$\frac{\partial J_{\mathrm{LQG}}}{\partial \boldsymbol{u}_{f}}=\mathbf{O}$

We obtain the minimized objective function's control rate: $u_{f}=-\left(\lambda \mathrm{W}_{u}+L_{u}^{T} \mathrm{~W}_{y} \mathrm{~L}_{u}\right)^{-1} L_{u}^{T} \mathrm{~W}_{y}\left(\mathrm{~L}_{w} \mathrm{~W}_{p}-r_{f}\right)$

By drawing LQG performance trade-off curve, whether there is room for improvement is evaluated.

\subsubsection{LQG benchmark implementation}

LQG performance benchmark takes the controlled variables' input and output variance into account. Actually, it provides an optimal bound that the present system can reach, the real controller only can be closer to it infinitely. The benchmark implementation steps are as follows:

(1) Collect the input and output parameters of current system over a period of time, and combine with statistical data, we can obtain the actual input $\left(J_{a c t-u}\right)$ and output $\left(J_{a c t-y}\right)$ variances.

(2) Taking advantage of the subspace modeling method, LQG control rate $\left(u_{f}\right)$ and predicted output $\left(y_{f}\right)$ can be calculated, and then we can obtain LQG benchmark $\operatorname{input}\left(J_{L Q G-u}\right)$ and $\operatorname{output}\left(J_{L Q G-y}\right)$ variance through further calculation.

(3) The input and output variances performance index on LQG benchmark:

Output variance index is as follows:

$\eta_{y}=\frac{J_{L Q G_{-} y}}{J_{a c t_{-} y}}$

Input variance index is as follows:

$\eta_{u}=\frac{J_{L Q G_{\_} u}}{J_{a c t \_u}}$

The control performance index is as follows:

$\eta=\frac{J_{L Q G}}{J_{a c t}}=\frac{J_{L Q G_{-} y}+J_{L Q G_{\_} u}}{J_{\text {act_y }}+J_{\text {act_u }}}$

\section{HIGH PRECISION FLIGHT SIMULATOR SYSTEM}

Actually, the flight simulator system is a typical high precision position/velocity servo system, by using the three frame motion simulator's angle, angle velocity, angle acceleration and other state changes, the flight attitude dynamic information are passed to the inertial navigation and guidance system, and after processing, the flight simulator can simulate aircraft's state.

The flight simulator is a three axis servo system, it has three relatively independent control channels, but the control structure of each channel is basically the same. Taking a three axis flight simulator as an example to analyze, we can build the entire structure diagram of the flight simulator servo system, as is shown in Figure 2: 


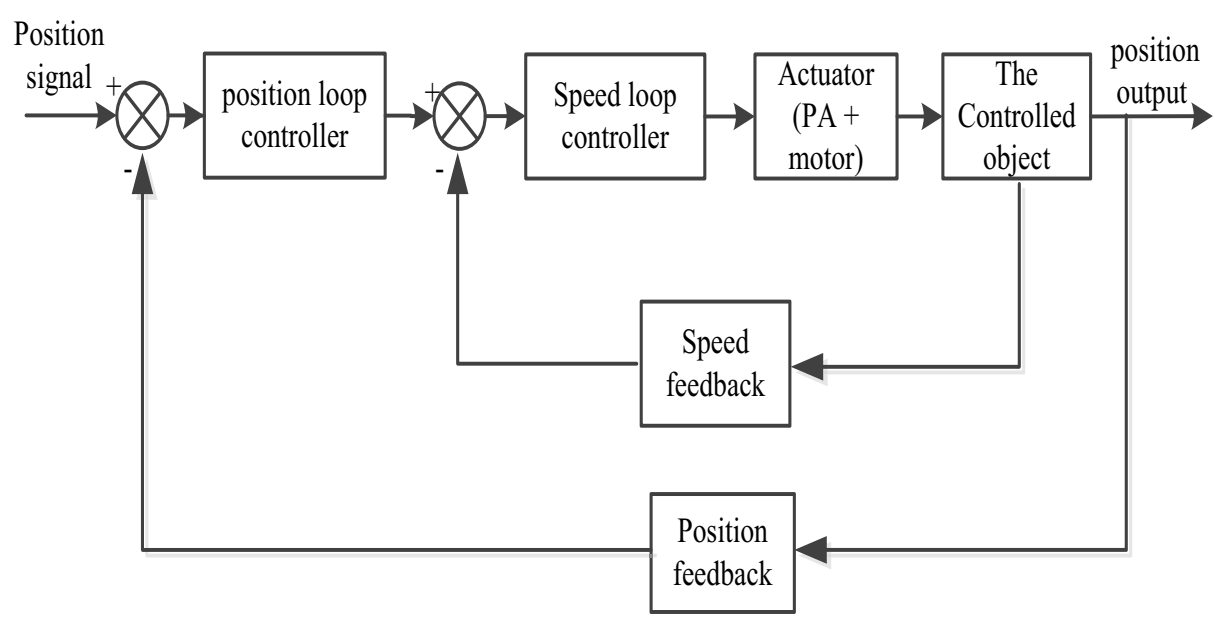

Figure 2. The structure diagram of the inner frame with friction model of the flight simulator servo system

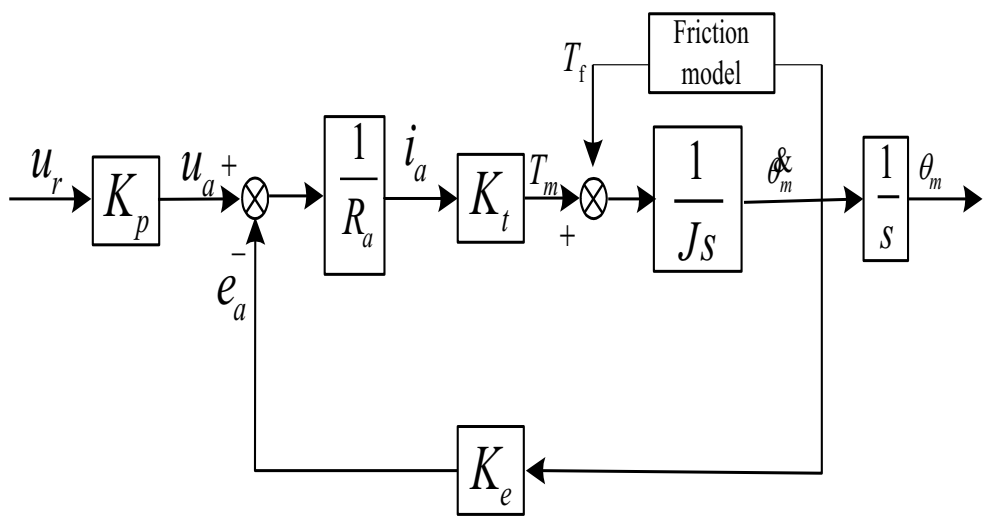

Figure 3. The structure diagram of the inner frame with friction model of the flight simulator servo system

In fact, any of its frame can be simplified as a linear two-order system, and the servo structure with friction model are shown in Figure 3.

Take $u_{r}(\mathrm{~V})$ as input voltage of the system, $R_{a}(\Omega)$ as the total resistance of the armature circuit, $K_{e}$ as voltage feedback coefficient, $K_{p}$ as the magnification of the power amplifier, $K_{t}$ as motor torque coefficient, $J$ as moment of inertia of the framework, $T_{f}$ as the friction torque, and $x_{1}(t)=\theta_{m}(t), x_{2}(t)=\dot{\theta}_{m}(t)$ According to Figure 3 , the state space equations of the flight simulator system which has friction model in inner frame can be described as:

$$
\left[\begin{array}{c}
\dot{x}_{1}(t) \\
\dot{x}_{2}(t)
\end{array}\right]=\left[\begin{array}{cc}
0 & 1 \\
0 & -K_{t} \frac{K_{e}}{J R_{a}}
\end{array}\right]\left[\begin{array}{l}
x_{1}(t) \\
x_{2}(t)
\end{array}\right]+\left[\begin{array}{c}
0 \\
K_{p} \frac{K_{t}}{J R_{a}}
\end{array}\right] u_{r}(t)-\left[\begin{array}{c}
0 \\
\frac{1}{J}
\end{array}\right] T_{f}(t)
$$

\section{HIGH PRECISION TURNTABLE SERVO SYSTEM CONTROLLER PERFORMANCE ASSESSMENT}

For the study of the turntable control algorithm, from the PID controller at the very beginning, controllers have been developed to advanced PID, fuzzy control, adaptive control, back stepping control, sliding model variable structure control and many other advanced controller. Each controller has its advantages and disadvantages.

Take the flight simulator system as the controlled object, and to evaluate the performance indexes of controllers of three kinds of algorithms. The parameters of a certain framework of flight simulator system are:

$$
\begin{aligned}
& \mathrm{R}_{a}=7.77 \Omega, \mathrm{K}_{t}=6 \mathrm{Nm} / \mathrm{A}, K_{e}=1.2 \mathrm{~V} /(\mathrm{rad} / \mathrm{s}), \\
& J=0.6 \mathrm{kgm}^{2}, K_{P}=11
\end{aligned}
$$

The Simulink simulation module is shown in Figure 
ICETA 2015

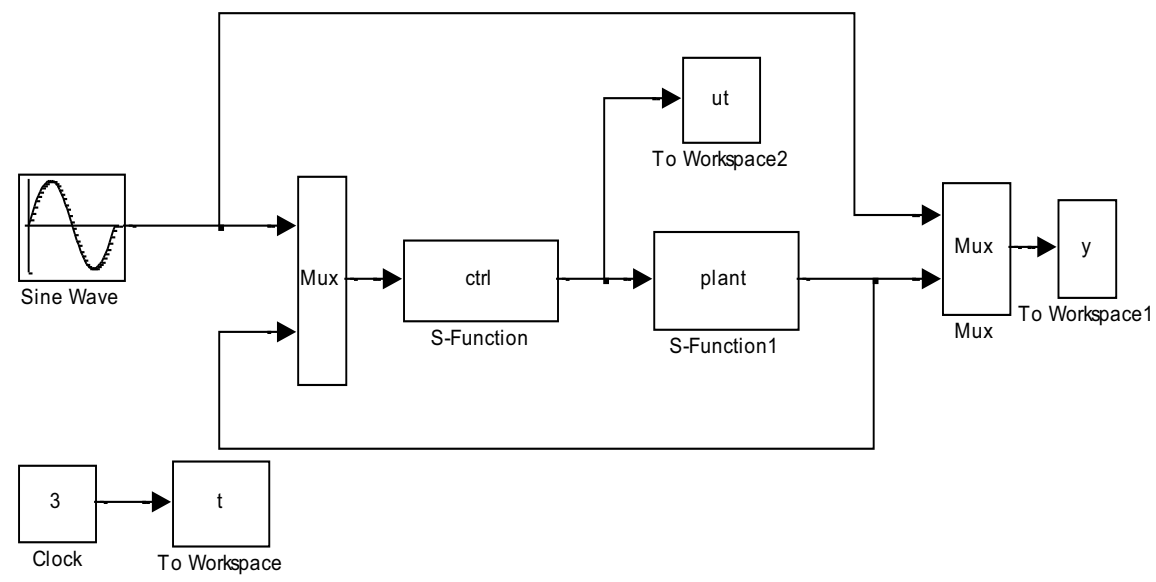

Figure 4. Inner frame of simulation module of the flight simulator servo system

To simulate in MATLAB/Simulink toolbox, selecting PID controller, sliding mode variable structure controller based on exponential approach law, an adaptive sliding mode controller for simulation of the controlled object, the position command signal is $r(t)=\sin (t)$, and the simulation time is set for $15 \mathrm{sec}-$ onds.

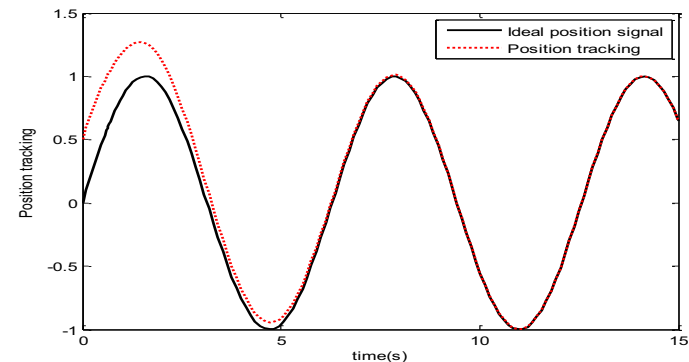

a

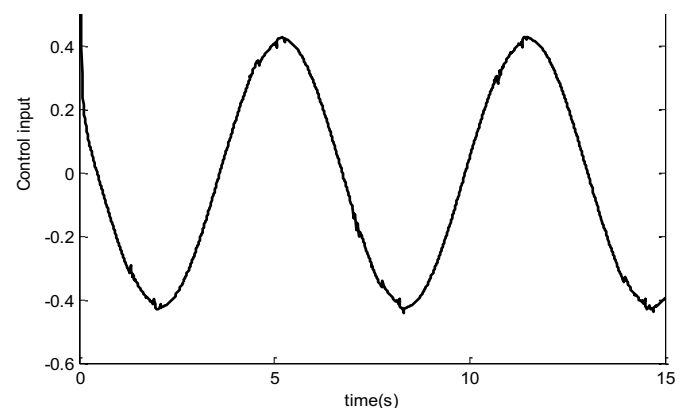

$\mathrm{b}$

Figure 5. The flight simulator servo system's position tracking curve and the control input curve in the control of PID controller

The simulation results are in Figures 5 7, which show the tracking curves and the control input signals in different controllers, where, black represents the input signal and the red represents output signal.
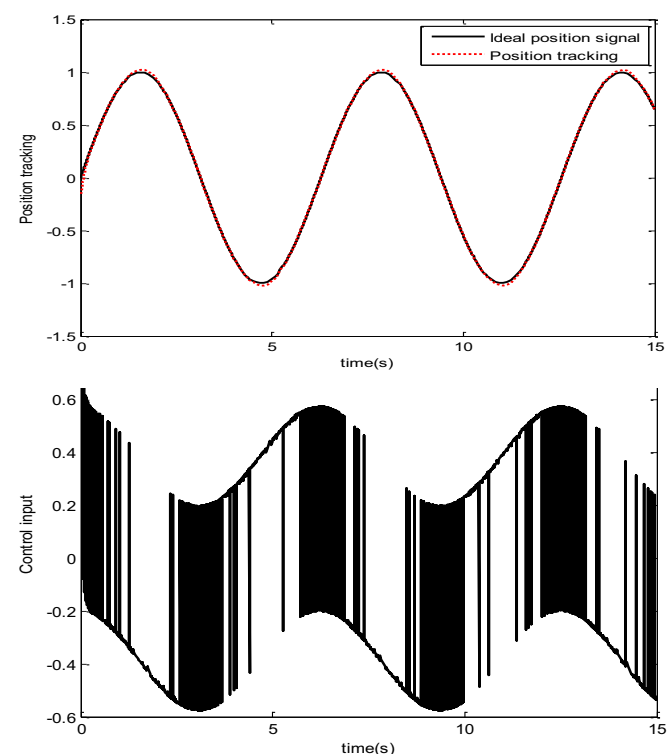

Figure 6. The flight simulator servo system's position tracking curve and control input curve under the control of the variable structure sliding mode controller based on exponential approach law

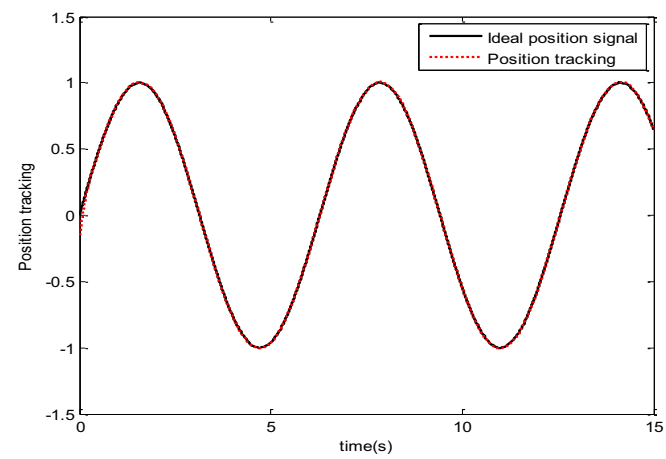




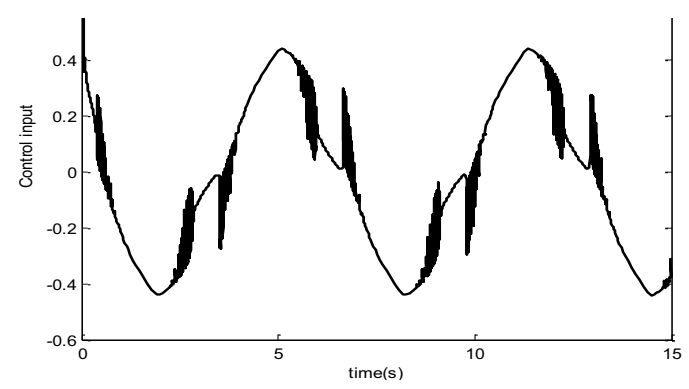

b

Figure 7. The flight simulator servo system's position tracking and control input curve in the control of adaptive sliding mode controller

The weight values $\lambda \in[0.1,100]$, and when $\lambda=0.1$, it is the minimum variance control, and the minimum output variance is $\eta_{y}=0.04$ when $\lambda=100$, it is the minimum cost control, and $\eta_{y}=0.14$. LQG curve are shown in Figure 8 .

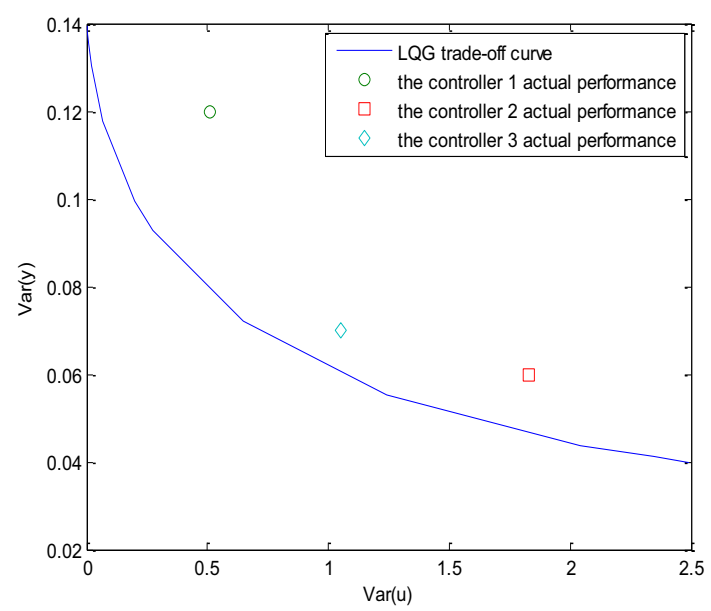

Figure 8. The LQG trade-off curve of the flight simulator servo system

The controller numbered 1 3 respectively represent PID controller, sliding mode controller based on exponent approaching law, and adaptive sliding mode controller.

LQG curve above is just a sketch; the performance of the actual controller is difficult to fully meet, and can only infinitely close. The specific problem is that we need to pass data to reflect the performance of calculation with the controller system.

Three different controller evaluation results are recorded in Table 1.

The corresponding optimal output variance of input variance of controller 1 is 0.08 , and then we can work out that $\eta_{y}=66.7 \%$, and $\eta_{u}=21.5 \%$. Through comparing, the input variance of controller 1 is the smallest, and the output variance is the largest. Even so, it is hard to decrease the input variance. If we want to achieve this goal, we need a more complex and optimizing PID controller, and we can improve the performance of the controller1 by minimizing the output variance. For controller $2, \eta_{y}=83.3 \%, \eta_{u}=66.7 \%$, and compared with controller 1 , the performance of controller 2 has been greatly improved. Especially the space of the output variance to enhance is very small, and for this kind of controller, we can improve its performance by reducing the input variance. For controller $3, \eta_{y}=85.7 \%, \eta_{u}=70.5 \%$. Obviously, the performance of controller 3 is better than controllers 1 and 2, and the comprehensive index $\eta_{L Q G 1} \leq \eta_{L Q G 2} \leq \eta_{L Q G 3}$ can prove that the performance index of the controller 3 is significantly better than the other two.

Table 1. Three kinds of controllers' LQG assessment results

\begin{tabular}{llll}
\hline & Controller1 & Controller 2 & Controller 3 \\
\hline $\operatorname{Var}(\mathrm{u})$ & 0.51 & 1.83 & 1.05 \\
$\operatorname{Var}(\mathrm{u})_{\mathrm{LQG}}$ & 0.11 & 1.22 & 0.74 \\
$\eta_{u}$ & $21.5 \%$ & $66.7 \%$ & $70.5 \%$ \\
$\operatorname{Var}(\mathrm{y})$ & 0.12 & 0.06 & 0.07 \\
$\operatorname{Var}(\mathrm{y})_{\mathrm{LQG}}$ & 0.08 & 0.05 & 0.06 \\
$\eta_{y}$ & $66.7 \%$ & $83.3 \%$ & $85.7 \%$ \\
$\eta_{L Q G}$ & $30.1 \%$ & $67.2 \%$ & $71.4 \%$ \\
\hline
\end{tabular}

The potential performance index can reflect the controller's improvable space compared with the optimal controller, and its expression is:

$P=\frac{J_{a c t}-J_{L Q G}}{J_{a c t}} \times 100 \%$

Accordingly we can calculate the potential performance of the three kinds of controllers which are $69.9 \%, 32.8 \%, 28.6 \%$. Obviously, potential performance index $P=1-\eta_{L Q G}$, meaning that the controller that has a better performance index has smaller space to improve. 


\section{CONCLUSION}

This paper gives an introduction for LQG performance evaluation method, takes the frame model of flight simulator servo system as an example and makes assessments on three different kinds of controllers, and then gives an improved direction. The simulation results indicate that we can estimate the flight simulator servo system's performance better by using LQG benchmark and analyze the potential performance index of each controller. It is conducive to the study of the development of flight simulator servo system and the research of the improvable method.

\section{ACKNOWLEDGEMENT}

This paper is sponsored by Natural Science Foundation of Inner Mongolia China (2014MS0611).

\section{REFERENCES}

[1] Harris T J. 1989. Assessment of closed loop performance. Canadian Journal of Chemical Engineering 67: 856-861.

[2] Huang B. \& Shah S L. 1999.. Performance Assessment of Control Theory and Applications. UK: Springer-Verlag.

[3] Kadali R. \& Huang B. 2002. Controller performance analysis with LQG benchmark obtained under closed loop conditions. ISA Transactions, 41(4): 521-537.

[4] Danesh N, Huang B, Shah S L. 2010. Performance assessment of advanced supervisory-regulatory control systems with subspace LQG benchmark. Automatic. 46: 1363-1368.

[5] Pour N D \& Shah S L. 2009. Consistency of noise covariance estimation in joint input-output closed-loop subspace identification with application in LQG benchmarking. Journal of Process Control, 19: 1649-1656. 